\title{
Budding insights on cell polarity
}

\author{
Dirk Fasshauer \& Reinhard Jahn
}

\begin{abstract}
The yeast proteins Sro7 and Sro77, and their metazoan relatives Lgl and tomosyn, interact with plasma membrane SNARE proteins and control cell polarity. Recently, the crystal structure of yeast Sro7 has been solved, yielding fascinating insights and even more unanswered questions concerning the molecular mechanism of these proteins.
\end{abstract}

Budding yeast is an attractive model organism for studying cell polarity. At the onset of asymmetric cell division, vesicles are transported along cytoskeletal tracks to a predefined site of the plasma membrane. Here, they undergo exocytosis, causing the formation of a bud that enlarges to form the daughter cell. A large octameric protein complex, termed the exocyst, has a key role in directing transport and defining the attachment site of the vesicles ${ }^{1,2}$. Fusion is mediated by the SNARE proteins Snc1/2, Sso1/2 and Sec9. Two homologous proteins, Sro7 and Sro77, bind both a component of the exocyst $(\text { Exo84 })^{3}$ and a SNARE protein $(\text { Sec9 })^{4}$. Deletion of these proteins leads to a cold-sensitive defect in vesicle exocytosis, suggesting a functional link between the exocyst and the SNAREs. Recently, Hattendorf et al..$^{5}$ have presented the crystal structure of a large portion of Sro7, shedding the first light on the molecular mechanism by which this protein may control SNARE function.

SNAREs are evolutionary conserved membrane proteins involved in all fusion events of the secretory pathway and are characterized by a conserved coiled-coil segment termed the SNARE motif. During fusion, the SNARE motifs of interacting SNAREs assemble into a parallel four-helix bundle that is initiated by the N-terminal tips and zips up toward the C-terminal membrane anchors. As a result, the membranes are pulled into close apposition and fusion is initiated. On the basis of a highly conserved set of amino acid side chains, SNARE motifs are classified into Qa-, Qb-, Qc- and R-SNAREs, and every functional SNARE complex contains one SNARE motif of each subfamily (reviewed in refs. 6,7). Sso1/2 (Qa), and Sncl/2 (R), each contain a single SNARE motif that is located adjacent to a C-terminal transmembrane domain. In contrast, Sec9 contains two SNARE motifs ( $\mathrm{Qb}$ and $\mathrm{Qc}$ ) connected by a linker region and has an extended $\mathrm{N}$-terminal domain.

Dirk Fasshauer and Reinhard Jahn are in the Department of Neurobiology, Max-PlanckInstitute for Biophysical Chemistry, Am Fassberg 11, 37077 Göttingen, Germany. e-mail:dfassha@gwdg.de and rjahn@gwdg.de
Sro proteins are large soluble proteins with close relatives in all eukaryotes. They contain multiple WD40 repeats of $\sim 40$ amino acid residues, typically containing a Trp-Asp pair at the carboxyl end. WD40 repeats are known to form blade-like structures that circularly assemble into $\beta$-propellers. A metazoan relative of Sro proteins, the tumor suppressor lethal giant larvae (Lgl), is important in establishing epithelial cell polarity during embryogenesis in Drosophila melanogaster ${ }^{8,9}$. The ensitivity to $\mathrm{NaCl}$ of a yeast Sro double mutant is rescued by ectopic expression of the Drosophila $\mathrm{lgl}$ gene $^{10}$. A mammalian ortholog of Lgl has been shown to interact with syntaxin-4 (ref. 11), a ubiquitous Qa-SNARE of the plasma membrane. The closest relative of Sro in animals is tomosyn, originally isolated as a binding partner of the Qa-SNARE syntaxin-1 (ref. 12). Syntaxin-1 mediates neuronal exocytosis, together with its SNARE partners synaptobrevin-2 (R) and SNAP-25 (Qbc). Thus, it appears that Sro, Lgl and tomosyn carry out similar functions by interacting with plasma membrane-specific Q-SNAREs.

The new crystal structure of Sro7 (ref. 5) shows that the 14 WD40 repeats fold into two consecutive seven-bladed $\beta$-propeller domains (Fig. 1a,b). The N-terminal portion of the chain associates with the C-terminal $\beta$-propeller to form a molecular 'clasp', yielding an overall topology similar to the actininteracting protein Aip1 (also called Unc78) ${ }^{13,14}$. The arrangement of the two $\beta$-propellers into an open clamshell-shaped structure provides multiple protein-binding surfaces oriented at specific angles with respect to one another. One of these surfaces is occupied by the 60-residue C-terminal tail of Sro7 (residues 892-951), which has an extended conformation that tightly packs against the convex surface of the $\mathrm{N}$-terminal $\beta$-propeller. A comparable arrangement has been found for the seven-bladed $\beta$-propeller proteins coronin-1 and Nup214 (also called CAN). In coronin-1, the tight binding of a 130-residue C-terminal tail stabilizes the $\beta$-propeller ${ }^{15}$, whereas in Nup214, the interaction is not needed to maintain the integrity of the $\beta$-propeller structure ${ }^{16}$. In the latter case, and probably also in Sro7, this interaction seems to be dynamic, suggesting that the C-terminal tail has a regulatory function.

Using the new structure as a guide, Hattendorf et al. ${ }^{5}$ have investigated how $\mathrm{Sec} 9$ binds $\mathrm{Sro} 7$ and how this interaction is related to the function of Sro7 in yeast exocytosis. They found that part of the N-terminal domain of Sec9 associates with the convex surface of the C-terminal $\beta$-propeller of Sro7. However, the region within the Sec $9 \mathrm{~N}$-terminal domain involved in the interaction is poorly conserved in other Sec9 proteins. Moreover, only fungal Qbc-SNARE proteins contain extended $\mathrm{N}$-terminal domains, and in yeast the $\mathrm{N}$-terminal domain of Sec9 has been shown to be expendable ${ }^{17}$.

To explore the observed discrepancy between the binding data and the functional complementation assays, the authors investigated whether the SNARE motifs of Sec9 alone are capable of interacting with Sro7. Weak binding was observed (with an approximate affinity of $50 \mu \mathrm{M}$ ), but only when the Sro7 tail was deleted. In addition, deletion of the tail helix moderately increased the affinity of full-length Sec9 (from 3 to $0.8 \mu \mathrm{M}$ ), suggesting that it regulates the ability of Sro7 to interact with Sec9. This gave rise to the hypothesis that the two SNARE motifs of Sec 9 compete with the tail of Sro for binding to the $\mathrm{N}$-terminal $\beta$-propeller. In this case, binding may control the ability of Sec9 to enter SNARE complexes. Further support for this notion will require competition experiments between the tail and the SNARE motifs of not only Sec9 but also its Qa-SNARE partner Sso, which might prove difficult, considering the low affinities.

Intriguingly, the tail region of Sro is followed by a stretch of C-terminal heptad-repeats (Fig. 1c,d), which are not included in the new crystal structure. This stretch was predicted to form part of a degenerate R-SNARE motif ${ }^{18}$, which is, in fact, less degenerate in the Sro7 orthologs of other fungi. Tomosyn (but not the Lgl proteins) contains a full R-SNARE motif at the C-terminal end ${ }^{19,20}$. The tomosyn R-SNARE motif is capable of substituting for synaptobrevin in forming a typical four 

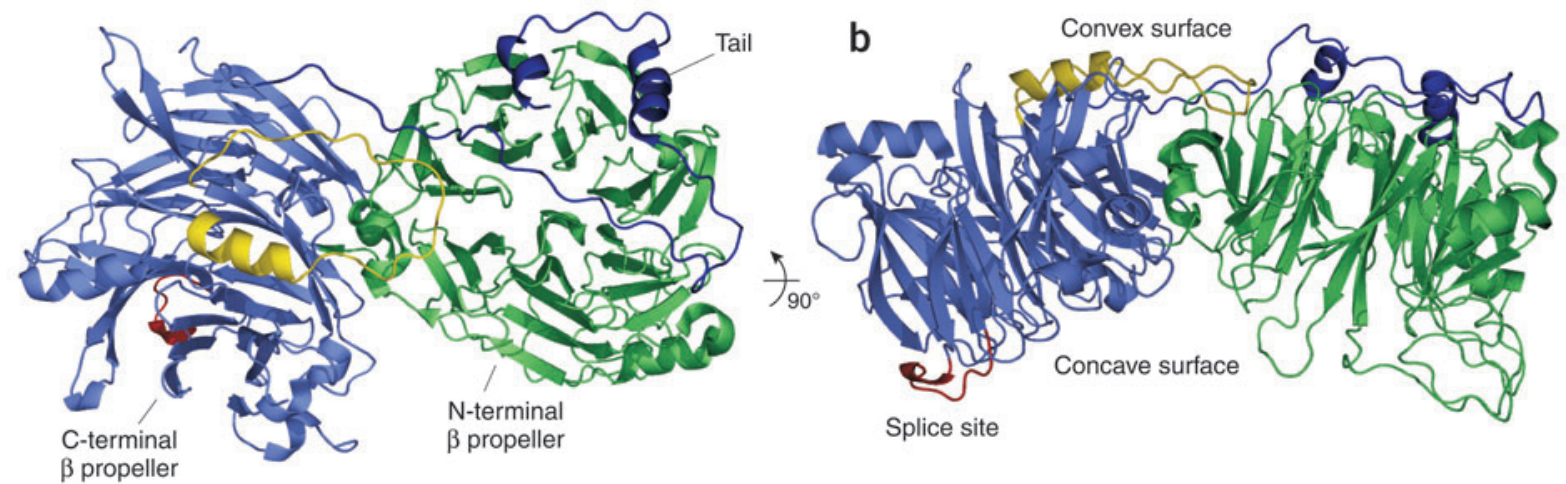

C

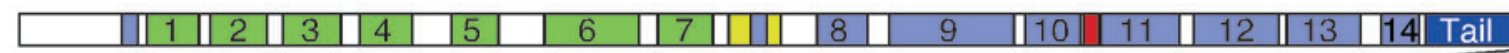

d

SaCe_Sro77p SaCe_Sro7p

MuMu_Syb2 RaNo_Tom1

DrMe Tom

CeEl_Tom

ArTh Tom

MaGr-Tom

NeCr Tom

AsNi-Tom

ScPo_Tom

$\Delta 42$

SSNAARKLPPGTKDHRYARPVRSSGRSNGYGVLKSVSRAIETRLDTVETTINDYATTMGQTMNDAMEETGRDMMKSAVGF FSERSSDDNNANHPEHQYTKPTRKGRNSSYGVLRNVSRAVETRWDAVEDRFNDYATAMGETMNEAVEQTGKDVMKGALGF

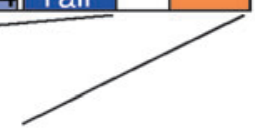

PNLTSNRRLQQTQAQVDEVVDIMRVNVDKVLERDQKLSELDDRADALQAGASQFETSAAKLKRKYWWKNLKMMIILGVICAIILIIIIVYFSS HIPGPG-GIEGVKGAASGVVGELARARLALDERGQKL SDLEERTAAMMSSADSFSKHAHEMMLKYKDKKWYQF HIPGPN--LEOLGORASTAASEISRAHOLAMERGEKLNLIEERAERMANTAODF SGTAHQLMLKYKDKKWYOI

TIPGPSVOMDRAOAGGVSAGQAAAMALONLNERTEKLNATVDATENL KNNAMSL SSRTGKLVEKYEKKKWYNF TIPGPSVQMDRAQAGGVSAGQAAAMALQNLNERTEKLNATVDATENL KNNAMSLSSRTGKLVEKYEKKKWYNF
PKTRTVDEIKSKYRKAGETSAIASQAKDKLHERGEKLERISORTAELQDNAENFASMAHELAKOMEKRKWWNI ARGGGVSSS TTAMRAGATQEGWGEYLTRQLNERTEKLNIVNDSMDNLQTKSQGWADDVNKYVSKQKKNLVLGAIKGKFS AAGAAGSSTARLQGQVKETEGWGDYLTRQLNERTEKLNIMGDSMDNLANTTSKWAEDVDGFVKKOKRDLFLGGIKKSFF

RRQAAREGRSTPARQNTNDEGYFAYMQRQVQERTEKLSFAGDNMDRLEETSSGWARDVNKYVQNQKKKAVLGVLGSKFGL SMTSQSAQSY IPPRRQQQQKGFFAQINDHLAQRGNMLGGIENTMDDLEEMSAEWANEIKDSLAGTKKDLILSGLKSYIP

$$
\begin{array}{llllllllllllllll}
-7 & -6 & -5 & -4 & -3 & -2 & -1 & 0 & 1 & 2 & 3 & 4 & 5 & 6 & 7 & 8
\end{array}
$$

e

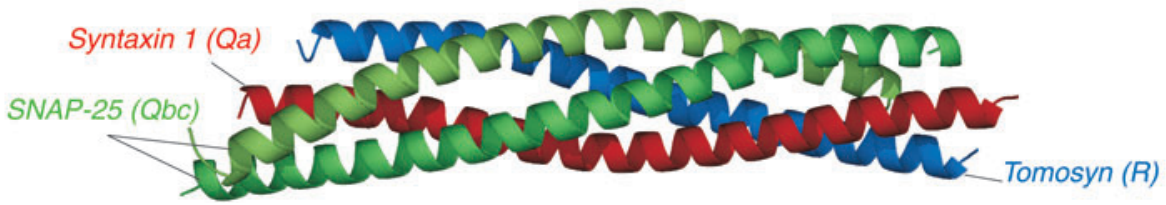

Figure 1 Schematic overview of the Sro7 and the tomosyn SNARE complex structures. (a) Convex surface of the Sro7 structure ${ }^{5}$. (b) Side view of a. (c) Schematic of the domain structure of Sro7, colored as in a,b. The region in red between blades 10 and 11 contains phosphorylation sites in animal tomosyn and $\mathrm{Lgl}$ and is alternatively spliced in animal tomosyns, thus probably providing distinct binding surfaces for different regulating factors. (d) Sequence alignment of C-terminal regions of tomosyns and synaptobrevin, showing that tomosyns generally contain an R-SNARE motif, which is partially degenerated in Saccharomycotina fungi. Shown are synaptobrevin-2 from Mus musculus (MuMu_Syb2, NCBI gene ID 207626) and tomosyn homologs from Rattus norvegicus (RaNo_Tom 1, 13540648), Caenorhabditis elegans (CaEl_Tom, 17508275), Drosophila melanogaster (DrMe_Tom, 28571171), Arabidopsis thaliana (ArTh_Tom1, 15239173), Magnaporthe grisea (MaGr_Tom, 39973855), Neurospora crassa (NeCr_Tom, 85090786), Aspergillus nidulans (AsNi_Tom, 40741778), Schizosaccharomyces pombe (ScPo_Tom, 19113918) and Saccharomyces cerevisiae (SaCe_Sro7p, 6325289; SaCe_Sro77, 6319362). Black highlight, synaptobrevin-2 transmembrane region; gray highlight, coiled-coil layer residues (numbers indicated at bottom). $\Delta 42$ marks position of deleted fragment ${ }^{5}$. (e) Structure of the SNARE complex of the C-terminal R-SNARE motif of tomosyn with the Q-SNAREs syntaxin-1 and SNAP-25 (ref. 18). Structure images were prepared with PyMOL (http://pymol.sourceforge.net).

helix-bundle with syntaxin-1 and SNAP-25 (Fig. 1e $)^{18}$. Thus, it appears that Sro7 and Sro77 are orthologs of tomosyn, with the two yeast isoforms probably arising from a recent gene duplication. Lgl, in contrast, is restricted to metazoans and probably has a role in cell differentiation in multicellular organisms. Apparently, an evolutionary split occurred between the two factors ${ }^{9}$, possibly resulting from an early duplication of the tomosyn gene in animals.

Considering that the R-SNARE motif of tomosyn can associate with the plasma membrane Q-SNAREs ${ }^{18}$, the question arises whether the degenerate SNARE motif in the yeast ortholog Sro7 can bind the R-SNARE site in the yeast exocytotic SNARE complex. In fact, the complex of Sro7 and Sec9 was originally isolated together with Sso and Snc. Hattendorf et al. ${ }^{5}$, however, did not observe a substantial change in binding affinity when the degenerate SNARE motif was deleted, although they did not test Sec9 binding in the presence of Sso. Furthermore, in complementation assays, a mutant Sro7 in which 42 residues were removed from the $\mathrm{C}$ terminus of the degenerate SNARE motif $(\Delta 42)$ was still able to rescue the Sro7/Sro77 deletion strain, whereas deletion of the entire tail region $(\Delta 142)$ was not tolerated.
Thus, neither of the two structurally defined interactions between Sro7 and the orthologous tomosyn and Q-SNAREs suffices to explain the function of these proteins at the molecular level. However, the new structure provides a sound basis for future experiments, and it is likely that the association of the tail peptide with the $\mathrm{N}$-terminal propeller domain will indeed prove to be a crucial element in the function of these fascinating proteins.

COMPETING INTERESTS STATEMENT

The authors declare no competing financial interests. 
1. Novick, P. et al. Biochem. Soc. Trans. 34, 683-686 (2006).

2. Munson, M. \& Novick, P. Nat. Struct. Mol. Biol. 13, 577-581 (2006)

3. Zhang, X. et al. J. Cell Biol. 170, 273-283 (2005).

4. Lehman, K., Rossi, G., Adamo, J.E. \& Brennwald, P. J. Cell Biol. 146, 125-140 (1999).

5. Hattendorf, D.A., Andreeva, A., Gangar, A. Brennwald, P.J. \& Weis, W.I. Nature 446, 567-571 (2007).

6. Hong, W. Biochim. Biophys. Acta 1744, 493-517 (2005)

7. Jahn, R. \& Scheller, R.H. Nat. Rev. Mol. Cell Biol. 7,
631-643 (2006).

8. Humbert, P.O., Dow, L.E. \& Russell, S.M. Trends Cell Biol. 16, 622-630 (2006)

9. Wirtz-Peitz, F. \& Knoblich, J.A. Trends Cell Biol. 16, 234-241 (2006).

10. Larsson, K. et al. J. Biol. Chem. 273, 33610-33618 (1998).

11. Fujita, Y. et al. Neuron 20, 905-915 (1998).

12. Musch, A. et al. Mol. Biol. Cell 13, 158-168 (2002).

13. Voegtli, W.C., Madrona, A.Y. \& Wilson, D.K. J. Biol. Chem. 278, 34373-34379 (2003).

14. Mohri, K., Vorobiev, S., Fedorov, A.A., Almo, S.C. \& Ono, S. J. Biol. Chem. 279, 31697-31707
(2004).

15. Appleton, B.A., Wu, P. \& Wiesmann, C. Structure 14 87-96 (2006).

16. Napetschnig, J., Blobel, G. \& Hoelz, A. Proc. Natl. Acad. Sci. USA 104, 1783-1788 (2007).

17. Brennwald, P. et al. Cell 79, 245-258 (1994).

18. Pobbati, A.V., Razeto, A., Boddener, M., Becker, S. \& Fasshauer, D. J. Biol. Chem. 279, 47192-47200 (2004).

19. Masuda, E.S., Huang, B.C., Fisher, J.M., Luo, Y. \& Scheller, R.H. Neuron 21, 479-480 (1998).

20. Fasshauer, D., Sutton, R.B., Brünger, A.T. \& Jahn, R. Proc. Natl. Acad. Sci. USA 95, 15781-15786 (1998).

\title{
Shifting nanoscopic clock gears
}

\author{
Susan S Golden, Vincent M Cassone \& Andy LiWang
}

\section{A recent report looks at a 'clock in a test tube' composed of the cyanobacterial proteins KaiA, KaiB and KaiC, revealing that these dancing proteins swap partners to keep track of time.}

In 2005, the research community was startled to learn that the cyanobacterial circadian clock is run by an oscillator that is, ironically enough, rather mechanical. This revelation came when Nakajima et al. ${ }^{1}$ built the oscillator in vitro with three proteins - KaiA, KaiB and $\mathrm{KaiC}$ - and ATP, and recapitulated the temperature-compensated circadian rhythm of phosphorylation of the KaiC oscillator protein. Now, several groups coordinated by C.H. Johnson have shown that, although evolution's watchmaker may be blind ${ }^{2}$, the oscillator itself, once built, can be seen in four dimensions. In this study by Mori et al. ${ }^{3}$, samples from an in vitro oscillating reaction were removed at intervals, imaged by electron microscopy and analyzed by two-dimensional electrophoresis to determine which partners are associated in a complex at different circadian times. Moreover, the authors present a mathematical model that incorporates known behaviors of the cyanobacterial circadian oscillator, accurately reproduces the rhythm of KaiC phosphorylation and formation of KaiABC complexes, and makes testable predictions about timekeeping states.

This contribution is the latest of several technical and conceptual leaps that have propelled the circadian clock of the humble cyanobacterium Synechococcus elongatus to fame, if not fortune, in two decades. In the first of these leaps, T.-C. Huang and colleagues demonstrated that daily rhythms in a number of physiological processes, reported by numerous

Susan S. Golden, Vincent M. Cassone and Andy LiWang are in the Center for Research on Biological Clocks, Texas A\&M University, College Station, Texas 77843, USA.

e-mail: sgolden@tamu.edu

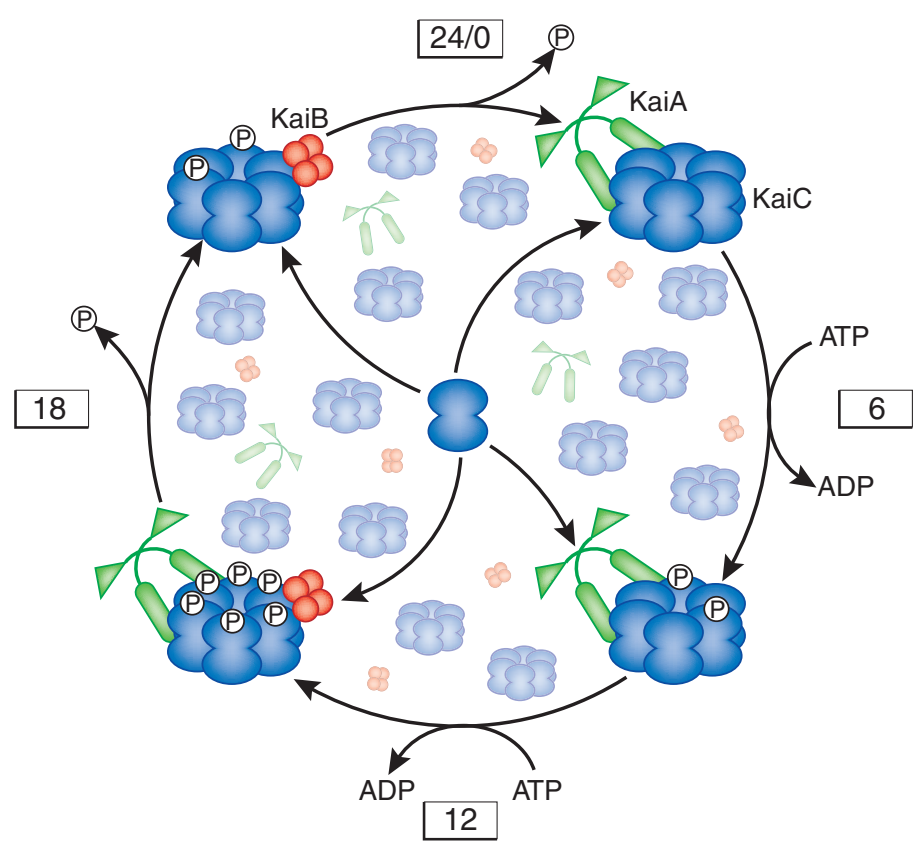

Figure 1 Schematic of the KaiABC central oscillator. Daily swings in KaiC phosphorylation are driven by rhythmic interactions with KaiA and KaiB. Although KaiA is found associated with KaiC throughout the circadian cycle, it enhances the autokinase activity of KaiC only from circadian time 0 hours (СT0) to CT12. Around CT12, KaiC becomes hyperphosphorylated, which induces a conformational change to a form with a higher affinity for KaiB. The KaiB-KaiC interaction results in an overall autophosphatase activity for KaiC, even in KaiB-KaiC-KaiA complexes. Once KaiC returns to its original hypophosphorylated state, at CT24, its conformation changes back to one with low affinity for KaiB, and the interaction between KaiA and KaiC starts the circadian cycle all over again. Interchange of KaiC monomers keeps rhythmic phosphorylation of KaiC particles phasecoherent across the population, which results in a robust ensemble-averaged amplitude for several cycles. Free KaiA, KaiB and KaiC are also present throughout the cycle.

researchers and in diverse cyanobacterial genera, have the characteristics of circadian rhythms as described for eukaryotic organisms: endogenous, self-sustained 24-hour rhythmicity in the absence of an externally applied cycle; ability to reset the relative timing of peaks to synchronize with relevant external environmental cues; and temperature compensation of the period ${ }^{4}$. Real progress in understanding the mechanism of the cyanobacterial circadian clock required an 